\title{
Influência de exercícios resistidos multiarticulares e monoarticulares na capacidade
}

\section{funcional do idoso}

\author{
Influence of multiarticular and monoarticular resistance exercises in the functional capacity of the
} elderly

Influencia de los ejercicios de resistencia multiarticulares y monoarticulares en la capacidad

funcional de las personas mayores

Recebido: 08/06/2021 | Revisado: 20/06/2021 | Aceito: 28/06/2021 | Publicado: 12/07/2021

Bárbara Borges Cançado e Lima ORCID: https://orcid.org/0000-0001-6965-0907 Centro Universitário Euro Americano, Brasil E-mail: barbara.borges@ hotmail.com

João Miguel de Andrade Mesquita Resende ORCID: https://orcid.org/0000-0002-3898-4368 Centro Universitário Euro Americano, Brasil

E-mail: joaomiguelresende@gmail.com

Denise Rodrigues Holsbach ORCID: https://orcid.org/0000-0003-2546-2378 Centro Universitário Euro Americano, Brasil E-mail: denisesartorelo@hotmail.com

Hudson Azevedo Pinheiro

ORCID: https://orcid.org/0000-0003-1081-4174 Secretária de Saúde do Distrito Federal, Brasil E-mail: hudsonap@gmail.com

Rafaello Pinheiro Mazzoccante ORCID: https://orcid.org/0000-0001-9901-8136 Centro Universitário Euro Americano, Brasil

E-mail: rafa_mazzoccante@hotmail.com

Frederico Santos de Santana ORCID: https://orcid.org/0000-0002-0766-6775 Universidade de Brasília, Brasil E-mail: fredericosantosdesantana@gmail.com

Leonardo Costa Pereira

ORCID: https://orcid.org/0000-0003-3319-5679

Centro Universitário Euro Americano, Brasil

E-mail: leonardo.pcllcp@gmail.com

\section{Resumo}

Com o passar dos anos ocorre o declínio da capacidade funcional do idoso. A capacidade funcional baseia-se em conseguir realizar as tarefas diárias de maneira autônoma, para tanto a inclusão de exercícios físicos resistidos no treinamento de idosos torna-se importante, visando analisar a eficácia do exercício multiarticular ou monoarticular no incremento da funcionalidade. Objetivo: Avaliar a influência do exercício resistido monoarticular e multiarticular no incremento da capacidade funcional do idoso. Método: Revisão integrativa da literatura realizada nos bancos de dados: PUBMED, LILACS e SCIELO, incluindo artigos científicos originais, nos idiomas português e inglês que versassem sobre treinamento resistido, capacidade funcional, exercício multiarticular e monoarticular em idosos. Determinou-se um coeficiente de eficiência em cada estudo selecionado. Resultados: Foram selecionados cinco artigos para compor a amostra, sendo que três fizeram utilização de exercícios multiarticulares em maior proporção dentro de seus protocolos de treinamento. Conclusão: Os exercícios multiarticulares parecem influenciar positivamente a capacidade funcional de idosos em detrimento dos exercícios monoarticulares, entretanto observou-se que o assunto é incipiente na literatura científica.

Palavras-chave: Idosos; Treinamento de força; Capacidade funcional.

\footnotetext{
Abstract

Introduction: Over the years, the elderly's functional capacity declines. Functional capacity is based on being able to carry out daily tasks in an autonomous way, so the inclusion of resistance physical exercises in the training of the elderly becomes important, aiming to analyze the effectiveness of multijoint or monojoint exercise in increasing functionality. Objective: To evaluate the influence of monojoint and multijoint resistance exercise in increasing the functional capacity
} 
of the elderly. Method: Integrative literature review carried out in the databases: PUBMED, LILACS and SCIELO, including original scientific articles, in Portuguese and English that dealt with resistance training, functional capacity, multijoint and monojoint exercise in the elderly. An efficiency coefficient was determined in each selected study. Results: Five articles were selected to compose the sample, three of which used multijoint exercises in greater proportion within their training protocols. Conclusion: Multijoint exercises seem to positively influence the functional capacity of the elderly to the detriment of monojoint exercises, however it was observed that the subject is incipient in the scientific literature.

Keywords: Seniors; Strength training; Functional capacity.

\section{Resumen}

Introducción: Con el paso de los años, la capacidad funcional de la persona mayor disminuye. La capacidad funcional se basa en poder realizar las tareas diarias de forma autónoma, por lo que cobra importancia la inclusión de ejercicios físicos de resistencia en el entrenamiento de las personas mayores, con el objetivo de analizar la efectividad del ejercicio multiarticular o monoarticular en el aumento de la funcionalidad. Objetivo: Evaluar la influencia del ejercicio de fuerza monoarticular y multiarticular en el aumento de la capacidad funcional del anciano. Método: revisión integradora de la literatura realizada en las bases de datos: PUBMED, LILACS y SCIELO, incluyendo artículos científicos originales, en portugués e inglés, que trataban sobre entrenamiento de resistencia, capacidad funcional, ejercicio multiarticular y monoarticular en ancianos. Se determinó un coeficiente de eficiencia en cada estudio seleccionado. Resultados: Se seleccionaron cinco artículos para componer la muestra, tres de los cuales hicieron uso de ejercicios multiarticulares en mayor proporción dentro de sus protocolos de entrenamiento. Conclusión: Los ejercicios multiarticulares parecen influir positivamente en la capacidad funcional del anciano en detrimento de los monoarticulares, sin embargo se observó que el tema es incipiente en la literatura científica.

Palabras clave: Anciano; Entrenamiento de fuerza; Capacidad funcional.

\section{Introdução}

O envelhecimento é um processo natural e progressivo em que ocorrem mudanças fisiológicas e funcionais. De acordo com Nogueira et al. (Nogueira et al., 2010), a capacidade funcional baseia-se nas habilidades físicas para realizar as atividades básicas da vida diária, é comum a diminuição da capacidade funcional no idoso, que se traduz pelo declínio de massa muscular, diminuição da capacidade coordenativa, agravando a mobilidade e perda da força muscular(Albin, Freitas, Teixeira, Gonçalves, \& Bós, 2006). Diminuindo o desempenho físico do idoso e contribuindo com a dependência para atividades diárias e risco de quedas (Campinas, Souza, \& Argento, 2010). Por tanto é de mera importância realizar avaliações para mensurar a capacidade funcional dos idosos, envolvendo a coordenação motora, força, agilidade, e equilíbrio (César, Mambrini, Ferreira, \& Lima-Costa, 2015).

A partir desse fato entende-se a relevância da inclusão de exercícios físicos para o tratamento eficaz na melhora da capacidade funcional do idoso, manutenção de sua saúde e qualidade de vida (Nathani Amorim Viana \& Clélia Maria de Sousa Ferreira Parreira, 2013). Por tanto o treinamento de força é recomendado de forma sistematizada e orientada por profissionais de Educação Física, melhorando ou preservando a força, manutenção da mobilidade articular e redução de riscos de quedas, levando a autonomia funcional dos idosos. Dessa forma o treinamento resistido é uma alternativa não farmacológica, aplicada as variáveis de treinamento, correspondendo ao volume, intensidade e frequência de treinamento (Pinheiro et al., 2018).

Maitin (Maitin, 2016) refere que no treinamento de força, os exercícios multiarticulares têm uma elevada ativação de grandes quantidades de músculos para a estabilização das articulações, assim recrutando mais unidades motoras que são responsáveis pela coordenação motora e como consequência a capacidade funcional do idoso. Vazquez et al. ${ }^{8}$ relatam que os exercícios monoarticulares podem corrigir assimetrias, disfunções mecânicas desenvolvidas no decorrer da vida. A hipótese do estudo é de que os exercícios multiarticulares serão mais eficientes para o aumento da capacidade funcional na população idosa.

A pergunta norteadora do presente estudo é: Quais exercícios resistidos entre multiarticulares e monoarticulares contribuem mais para o aumento da capacidade funcional em idosos?

Sendo assim, o objetivo do presente trabalho é verificar a influência do treinamento resistido multiarticular e monoarticular na capacidade funcional do idoso. 


\section{Método}

Pesquisa de revisão integrativa de literatura, com busca sistematizada (Galvão \& Pereira, 2014). Foi realizada a análise de artigos referentes ao treinamento de força em população idosa, nos idiomas português e inglês, nas seguintes bases de dados: SCIELO, PUBMED e LILACS. A coleta foi realizada no mês de setembro de 2018.

Como critérios de inclusão utilizaram-se os descritores referentes ao treinamento resistido, capacidade Funcional, exercício multiarticular e monoarticular em idosos. Os critérios de exclusão foram artigos que versassem sobre treinamento resistido com fins terapêuticos, patologias, lesões, treinamento em animais, estudos de revisão bibliográfica, teses, ausência de testes funcionais e protocolos de treinamento (intensidade, frequência semanal, tipos de exercícios).

No banco de dados PUBMED, foram utilizados os descritores oficiais: multijointand strenght training and elderly $\mathrm{e}$ Onejointand strenght training and elderly and functional. No banco de dados Scielo e Lilacs, foram utilizados os descritores oficiais: Treinamento de Força em idosos e Capacidade Funcional.

Para analisarmos o percentual de melhora da capacidade funcional nos artigos, utilizaremos o Delta como referência, entre as avaliações funcionais pré e pós-treinamento, como no seguinte cálculo: Avaliação Pré - Pós = Melhoria.

Quando o resultado for negativo, deve ser multiplicado por (-1), para os testes que o Pós seja positivo em relação ao Pré.

$$
\Delta=\frac{\text { AvaliacãoPré }}{\text { Melhoria }}=\frac{100 \%}{X}
$$

Para comparar os exercícios multiarticulares e monoarticulares utilizaremos a seguinte equação.

$$
\text { (R.S). (I) } .(\mathrm{F}) \cdot(\mathrm{T}) \cdot(\mathrm{E})=\frac{\Delta \%}{\mathrm{CT}}=\mathrm{CE}
$$

A avaliação do Coeficiente de Eficiência (CE) dos exercícios, será dado através dos dados citados acima em cada artigo do estudo.

Repetições (R) multiplicado pelas séries (S), multiplicado pela intensidade (I), multiplicado pela frequência semanal de treinamento $(\mathrm{F})$, multiplicado pelo tempo total do estudo $(\mathrm{T})$ e pelo número de exercícios $(\mathrm{E})$, resultando no coeficiente de trabalho $(\mathrm{CT})$.

O Delta \% será o resultado dos testes avaliados nos artigos selecionados, na qual o CT será dividido pelo Delta \% resultando no coeficiente de eficiência (CE).

Será evidenciado através de porcentagem os exercícios analisados em cada artigo entre multiarcular e monoarticular.

$$
\underline{\mathrm{NE}}=\underline{100 \%}
$$

\section{NEM $X$}

Então o Coeficiente de Eficiência estará junto com o percentual dos exercícios analisados em determinado artigo, para analisarmos se os exercícios multiarticulares obtiveram efeito na eficiência do artigo analisado. 


\section{Resultados}

Nas consultas preliminares para identificar os artigos relacionados aos exercícios multiarticulares e monoarticulares no treinamento de força que visam à melhoria da capacidade funcional do idoso, foram encontradas 140 publicações nos bancos de dados (PUBMED, SCIELO e LILACS) com temas similares.

Ao analisar as publicações quanto aos descritores oficiais, foi verificado que:

- $\quad$ PUBMED -Multijointand, strenght training and elderly - possuia 19 artigos, sendo 2 selecionados arbitrariamente para amostra. Ao avaliar o resumo dos estudos, verificou-se que nenhum estava apto a ser utilizado para atender os objetivos desta pesquisa. Os critérios de exclusão foram "revisão de literatura", "patologias/lesões", "treinamentos em não idosos";

PUBMED -Onejointand strenght training and elderly and functional - possuia 52 artigos, dos quais 8 foram selecionados aleatoriamente para amostra. Apenas 3 foram aproveitados, pois os demais foram excluídos devido à "revisão de literatura", "população não idosa", "treinamento não resistido" e "ausência de testes funcionais";

- SCIELO - treinamento de força em idosos, capacidade funcional - possuía 19 artigos, sendo 4 selecionados aleatoriamente para amostra. Ao avaliá-los, foram empregados 2, e os outros 2 foram excluídos por "não conter variável de intensidade" e outro por se tratar de "fins terapêuticos", e "Treinamentos em não humanos".

LILACS - treinamento de força em idosos, capacidade funcional - possuía 51 artigos, dos quais 9 foram selecionados arbitrariamente para amostra, entretanto todos os estudos foram suprimidos por "repetição de publicação", "ausência de exercícios", "ausência de testes funcionais".

O Fluxograma 1 resume a análise:

Fluxograma 1 - Análises da pesquisa.

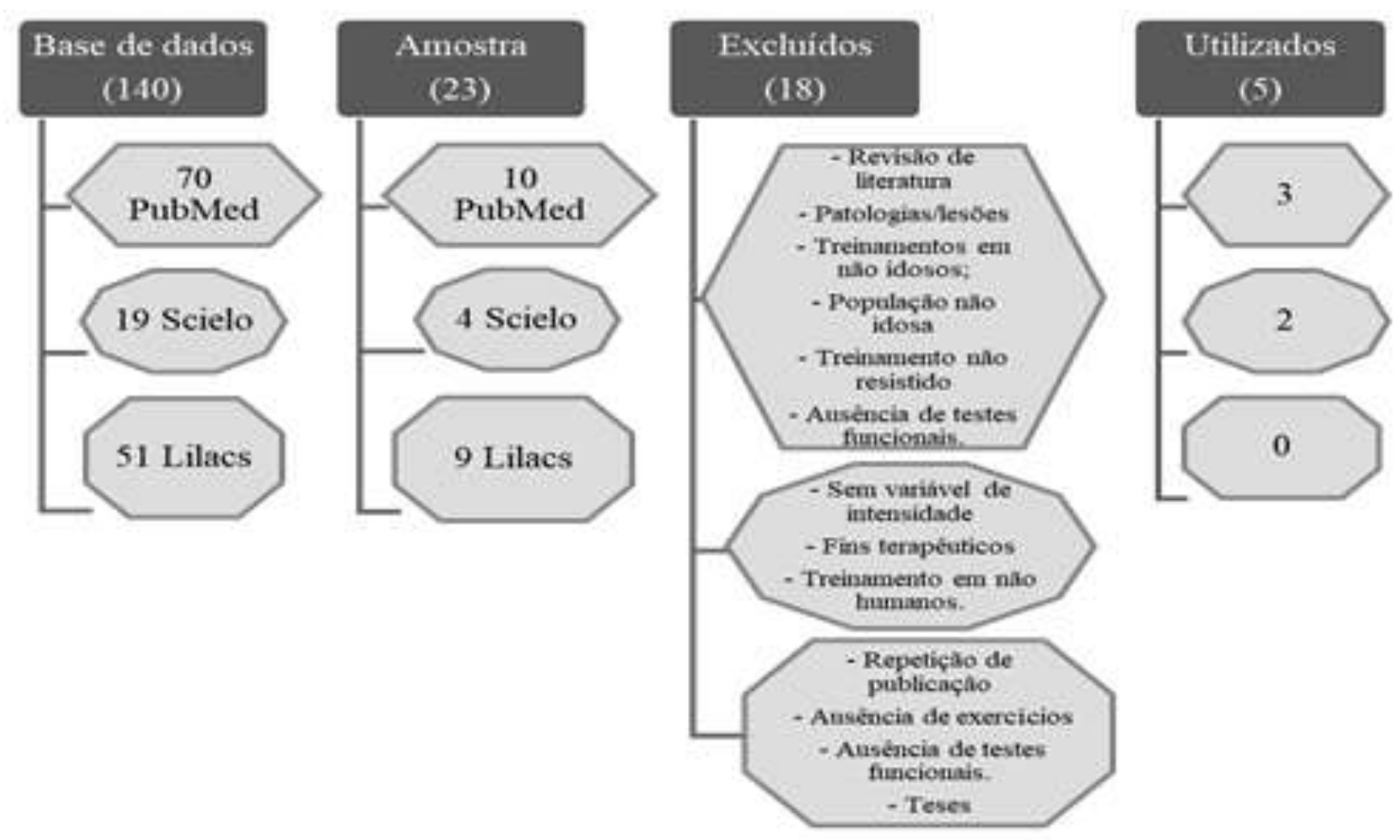

Fonte: Autores. 
O quadro a seguir, contém os resultados de acordo com as referências extraídas dos artigos:

Quadro 1 - Resultados da pesquisa.

\begin{tabular}{|c|c|c|c|c|c|c|c|}
\hline AUTOR & AMOSTRA & TESTES & SEMANAS & $\Delta(\%)$ & $\begin{array}{l}\text { EXERCÍCIOS } \\
\text { Multiarticulares }\end{array}$ & $\mathrm{CT}$ & CE .(1000) \\
\hline $\begin{array}{l}\text { Lustosa et al., } \\
\text { (2011) }\end{array}$ & $\begin{array}{l}\text { Feminino } \\
>65 \text { anos }\end{array}$ & TUG; TC 10. & 10 & $8 \%$ & $14 \%$ & 378.000 & 0,02116 \\
\hline Neto et al.,(2017) & $\begin{array}{c}\text { Feminino } \\
(65.6 \pm 4.6) \\
\text { Anos }\end{array}$ & TUG,TSL,TFC & 8 & $20 \%$ & $75 \%$ & 307.200 & 0,06510 \\
\hline Tracy et al., (2005) & $\begin{array}{c}\text { Masculino } \\
(72.0 \pm 4.6) \\
\text { Anos }\end{array}$ & $\begin{array}{c}\text { TSE,TDE,TSL, } \\
1 \mathrm{RM}\end{array}$ & 16 & $2,8 \%$ & $0 \%$ & 86.400 & 0,03241 \\
\hline $\begin{array}{l}\text { Kobayashi et } \\
\text { al.,(2012) }\end{array}$ & $\begin{array}{c}\text { Feminino } \\
\text { Masculino } \\
(67.5 \pm 5.3) \text { anos }\end{array}$ & TSL,TDE,TSE,TEP & 8 & $36 \%$ & $57 \%$ & 453.000 & 0,07937 \\
\hline Orssattoetal.,(2017) & $\begin{array}{c}\text { Feminino } \\
\text { Masculino } \\
(63.5 \pm 2.6) \text { anos }\end{array}$ & $1 \mathrm{RM}$ & 12 & $87 \%$ & $100 \%$ & 48.600 & 1,79012 \\
\hline
\end{tabular}

Legenda: Timed Up andGo -TUG; Teste de Caminhada de 10 min - TC 10; Teste de Sentar e Levantar - TSL; Teste de Flexão de Cotovelo - TFC; Teste de Subida de Escada - TSE; Teste de Descida de Escada - TDE; Teste de Força Máxima - 1RM; Teste de Equilíbriona Plataforma - TEP; Melhoria- $\Delta(\%)$;Coeficiente de Trabalho - CT; Coeficiente de Eficiência - CE.

Fonte: Autores.

Quadro 2 - Protocolos de treinamento.

\begin{tabular}{|c|c|c|c|c|c|}
\hline $\begin{array}{c}\text { PROTOCOLO } \\
\text { TREINAMENTO }\end{array}$ & EXERCÍCIOS & SÉRIES & REPETIÇÕES & INTENSIDADE & $\begin{array}{l}\text { FREQUÊNCIA } \\
\text { SEMANAL }\end{array}$ \\
\hline $\begin{array}{l}\text { Lustosa et al., } \\
\text { (2011) }\end{array}$ & $\begin{array}{l}\text { Flexão, Extensão, Abdução, Adução do quadril; } \\
\text { flexão, extensão do joelho; e agachamento. }\end{array}$ & 3 & 8 & $75 \% 1 \mathrm{RM}$ & $3 X$ \\
\hline $\begin{array}{l}\text { Neto et al., } \\
(2017)\end{array}$ & $\begin{array}{l}\text { Agachamento no smtih, legpress } 45^{\circ} \text {, stiff, supino } \\
\text { máquina, remada, mesa flexora, puxada frontal, } \\
\text { flexão plantar. }\end{array}$ & 2 & 10 & $80 \%$ OMNI-GSE & $3 X$ \\
\hline Tracy et al., (2005) & Extensão de joelho unilateral;bilateral. & 3 & 10 & $30 \% 1 \mathrm{RM}$ & $3 X$ \\
\hline $\begin{array}{l}\text { Kobayashi et al., } \\
\text { (2012) }\end{array}$ & $\begin{array}{l}\text { Leg Press; Puxada frontal; Cadeira Adutora; Pull } \\
\text { over; Triceps Paralela (máquina); Crucifixo } \\
\text { (máquina); Extensão de quadril (graviton) }\end{array}$ & 6 & 15 & $30 \% 1 \mathrm{RM}$ & $3 X$ \\
\hline $\begin{array}{l}\text { Orssatto et al., } \\
\text { (2017) }\end{array}$ & Legpress $45^{\circ}$ & 3 & 9 & $75 \% 1 \mathrm{RM}$ & $2 \mathrm{X}$ \\
\hline
\end{tabular}

Fonte: Autores.

Os anos de publicação dos estudos selecionados vão desde 2005 a 2017, englobando uma amostra heterogênea da população idosa. Há variações dos testes funcionais, no entanto os mais utilizados foram, o Timed Up and Go (TUG), Sentar e Levantar (TSL), força máxima (RM) e Subida e Descida de escada (TSE, TDE). Houve mensuração das capacidades de potência, força, agilidade, equilíbrio e resistência. 
O editorial de Orssatto et al.(Orssatto, Moura, Sakugawa, Radaelli, \& Diefenthaeler, 2017) destacou-se por ter o maior percentual de melhoria, correspondendo à $87 \%$ do coeficiente delta, utilizando $100 \%$ de presença de exercícios multiarticulares. Apresentando um coeficiente de eficiência superior aos demais. Além disso, outros dois estudos de Kobayashi et al.(Kobayashi, Koyama, Enoka, \& Suzuki, 2012) e Resende Neto et al. (Neto, Santos, Silva, Santana, \& Silva-Grigoletto, 2018), observou-se o emprego de exercícios multiarticulares acima de 50\%, tendo em vista a aparição de melhores coeficientes de eficiência.

Lustosa et al. (Lustosa et al., 2011) e Tracy e Enoka (Tracy \& Enoka, 2006) apresentaram resultados abaixo de 50\% e ausência de exercícios multiarticulares, tiveram valores inferiores em relação ao coeficiente de eficiência comparado aos outros estudos. No entanto Tracy et al.(Tracy \& Enoka, 2006) apresenta um valor inferior de coeficiente de trabalho e um valor superior de coeficiente de eficiência, quando comparado com Lustosa et al. (Lustosa et al., 2011), pois em seu protocolo de treinamento evidencia-se a baixa intensidade; já no protocolo de treinamento de Lustosa et al. (Lustosa et al., 2011), a intensidade é elevada.

\section{Discussão}

Diante dos resultados encontrados, especula-se que a presença de exercícios multiarticulares no treinamento resistido possam contribuir com mais eficiência que os exercícios monoarticulares para a funcionalidade dos idosos. O estudo que alcançou maior coeficiente de eficiência, expondo esse achado é a publicação de Orssatto et al.(Orssatto et al., 2017), no qual propões protocolo de treinamento com a utilização de um único exercício multiarticular, o Legpress $45^{\circ}$. Nos estudos de Gentil et al.(Gentil, Soares, \& Bottaro, 2015) bem como Fleck e Figueira (Fleck \& Figueira Junior, 2003) afirmam que ao se realizar exercícios multiarticulares todos os grupamentos musculares diretamente envolvidos são adequadamente estimulados, obtendose os mesmos ganhos de força e massa muscular que se consegue com os exercícios de isolamento. Nessa perspectiva presumese que os exercícios multiarticulares exigem maior coordenação neural entre os músculos. No entanto, por se envolver em mais de uma articulação e mais de um grupo muscular na execução do movimento, implica-se um aprendizado motor mais demorado e complexo, já nos exercícios monoarticulares por se envolver apenas em uma articulação, resultam em um movimento mais simples e fácil para o aprendizado motor. Ainda assim os exercícios multiarticulares alcançam maior trabalho de grupamentos musculares ao mesmo tempo, assim sendo favoráveis em termos funcionais.

Já nos resultados de coeficiência de trabalho expõe ser inferior aos demais estudos, acreditando que o protocolo de treinamento apresenta uma frequência semanal menor que os demais, totalizando duas vezes por semana, porém com uma elevada intensidade, utilizando 75\% de uma repetição máxima. De acordo com Bertani et al.(Bertani, Campos, \& Patrizzi, 2014), o treinamento resistido de alta intensidade, sendo bem aceitos na literatura são valores maiores ou iguais a 75\% de uma repetição máxima, nesta população. Gentil et al.(Gentil et al., 2015) relatam que ao se utilizar exercícios multiarticulares em intensidades altas, todos os músculos envolvidos trabalharam intensamente e serão adequadamente estimulados. Evidenciando assim um pretexto para uma frequência semanal ser menor, e devido a população do estudo ser composta por idosos, o tempo de recuperação necessária é maior, devido à diminuição progressiva da capacidade fisiológica de desempenho(Mcardle, Katch, \& Katch, 1998).

Os testes que foram mensurados para a capacidade funcional dos idosos, foi meramente o teste de força máxima (RM), no qual obtiveram valores de melhoria superiores equiparando aos estudos dos distintos autores. O teste de uma repetição máxima de acordo com Bertani et al. (Bertani et al., 2014) é subjugado por mensurar a capacidade funcional de força. No entanto o teste de força máxima realizado em pesquisa de Coelho (Coelho, Souza, Roncada, Tiggemann, \& Dias, 2014) não mensura como um todo o desempenho da capacidade funcional, ou seja, que a capacidade funcional é pouco influenciada somente pela força máxima, mas pelo condicionamento físico de uma forma geral. Essa ação funcional requer a atuação de outras capacidades físicas como, equilíbrio, agilidade e cardiorrespiratório. Estudo de Roncato et al.(Roncato, Galaza, Freire, Tiggermann, \& Dias, 2014) desenvolvido em idosas, foi observado que a produção de força nos testes de 1RM em dois exercícios de membros inferiores 
(extensão de joelhos e legpress) não apresentou correlação significativa com o desempenho funcional. Todavia, a perda de força e massa muscular durante o envelhecimento está diretamente relacionada com a perda da capacidade funcional do idoso tendo em vista que a força é uma valência física de suma importância para desempenhar as atividades de vida diária(Bertani et al., 2014; Coelho et al., 2014; Lima et al., 2012; Roncato et al., 2014).

Pesquisa realizada por Kobayashi et al.(Kobayashi et al., 2012) expõe em um protocolo de treinamento exercícios multiarticulares e monoarticulares bem distribuídos, em que 57\% são multiarticulares, atingindo o segundo maior coeficiente de eficiência, tendo o mais alto coeficiente de trabalho, referente aos outros autores. É evidenciado que o aumento significativo do coeficiente de trabalho está correlacionado ao protocolo de treinamento, totalizando em um volume maior, com maiores números de repetições e séries, utilizando apenas 30\% de uma repetição máxima. A população heterogênea de idosos do estudo não possuíam a prática de treinamento de força, especulando-se que o uso de uma intensidade mais baixa está relacionado a esse fator.

Nasser e Corrêa-Neto (Nasser \& Neto, 2017)referem que em treinamentos com baixa intensidade há bons resultados nas adaptações musculares, sendo menos exaustivos, podendo ser uma boa estratégia para evitar lesões, já que em cargas mais elevadas as sobrecargas nas articulações são maiores quando comparados com os treinos de carga reduzida. Segundo Araújo et al.(Lorenzi, Araújo, Fló, \& Muchale, 2010), o treinamento resistido com menor intensidade também beneficia o desempenho funcional dos idosos, especialmente em sedentários.

Nas avaliações dos testes complexos (Sentar e Levantar; Descer escada; Subir escada) e de equilíbrio, se observou melhora na capacidade funcional de $36 \%$, em detrimento das pesquisas de Lustosa et al. ${ }^{12}$ e Tracy e Enoka (Tracy \& Enoka, 2006), no qual apresentaram resultados inferiores a $10 \%$ de melhoria. Nota-se variações dos testes avaliados nos quesitos mobilidade articular, agilidade, potência, cardiorrespiratória e força de membros inferiores, apresentando desempenhos positivos em relação aos testes funcionais com as capacidades físicas citadas. No estudo de Silva et al. (Silva, Pazzinatto, \& Albuquerque, 2014), os testes mencionados intervêm na diminuição de riscos de quedas, proporcionando maior qualidade funcional e autonomia do idoso.

A pesquisa desenvolvida por Tracy e Enoka (Tracy \& Enoka, 2006) apresenta em seu protocolo de treinamento dois exercícios monoarticulares. Desta forma, ocorreu pouco incremento na capacidade funcional, relacionado aos testes funcionais (Sentar e Levantar; Descer escada; Subir escada e Força máxima). Tal protocolo de treinamento destaca-se pela baixa intensidade, utilizando 30\% de uma repetição máxima, apresentando um valor inferior de coeficiente de trabalho e um valor superior de coeficiente de eficiência, quando comparado com Lustosa et al.(Lustosa et al., 2011). Dessa forma, se pressupõe que o método usado no treinamento, pode influenciar nos valores do coeficiente de eficiência. O método utilizado no estudo foi o método com isometria conjugado com movimentos dinâmicos em repetições consecutivas, favorecendo em executar repetições forçadas devido a fadiga atingida. Por ser um treino metabólico, as respostas são provindas das alterações metabólicas que de acordo com Frois e Paulo (Das et al., 2011)e Gentil et al.(Gentil et al., 2015) estão associadas as mudanças de aumento das concentrações de GH, Lactato, Cortisol e fatores de crescimento locais como IGF-1, favorecendo em ganhos de força e hipertrofia. E repetições forçadas são capazes de impor maior carga ao músculo do que métodos tradicionais, gerando também maior quantidade de microlesões, possuindo eficácia superior a métodos tradicionais.

O estudo de Lustosa et al.(Lustosa et al., 2011), em seu protocolo de treinamento executou maior número de exercícios monoarticulares, envolvendo a presença de $14 \%$ de multiarticulares, tendo um valor de melhoria superior, através dos resultados dos testes funcionais, que foram distintos se comparados aos testes de Tracy e Enoka(Tracy \& Enoka, 2006), utilizando o teste Timed Up and Go e da Caminhada de 10 minutos, avaliando as capacidades físicas de equilíbrio dinâmico, agilidade e cardiorrespiratório. Porém, o coeficiente de eficiência é menor, levando em consideração que o coeficiente de trabalho é maior. E a perda de força está relacionada com a idade, por tanto é importante mensurar essa valência física. Segundo Fleck e 
Figueira(Fleck \& Figueira Junior, 2003), a perda de força pode ser causada por fatores miogênicos, em que a diminuição de massa muscular incide mais sobre as fibras tipo II, aumentando assim a concentração das fibras tipo I e redução na área de secção transversa. Dessa forma, idosos possuem uma predisposição à atrofia nas fibras tipo II, e Coggan et al.(Coggan et al., 1992) corroborando completa, relatando que tal fato é pior no sexo feminino.

A amostra do estudo também pode estar associada ao coeficiente de eficiência, por apresentar uma amostra de homens ativos e com maior tempo de aplicação enquanto a amostra de Lustosa et al.(Lustosa et al., 2011) são de idosas frágeis e com tempo de aplicação cerca de seis semanas menor.

No entanto em seu protocolo de treinamento há uma intensidade mais elevada, de 75\% de uma repetição máxima, com uma amostra de idosas frágeis, que está correlacionada a diminuição na velocidade da marcha, níveis baixos de atividade física e diminuição de força muscular. A sarcopenia é muito comum para esse tipo de população(Lustosa et al., 2011). Então quando comparado os dois estudos, pode-se perceber que ambos aparentam ser ineficientes, devido à escassez de exercícios multiarticulares ou ausência dos mesmos no protocolo de treinamento.

Na pesquisa de Resende-Neto et al. ${ }^{11}$ predominam os exercícios multiarticulares no protocolo de treinamento, sendo $75 \%$, tendo o segundo maior porcentual de presença dos multiarticulares, comparado aos demais. Porém apresenta-se ter um coeficiente de eficiência menor que o estudo de Kobayashi et al. (Kobayashi et al., 2012), que utilizou 18\% a menos de exercícios multiarticulares. Esse fato pode ser explicado por ter uma amostra de idosas sedentárias, não praticantes de exercícios físicos. Todavia ambas as publicações tiveram o mesmo tempo de estudo, respectivamente 8 semanas. Nas avaliações dos testes, há um que se difere dos demais estudos, no qual foi utilizado o teste de flexão de cotovelos, um teste que possui uma análise para a verificação da resistência muscular localizada de membros superiores. No entanto os testes de Timed Up and Go, Sentar e Levantar, foram utilizados por muitos autores em suas pesquisas, devido a confiabilidade que esses testes trazem para avaliação de controle postural, diagnóstico dos preditores do risco de quedas, avaliando a mobilidade, equilíbrio, flexibilidade das articulações dos membros inferiores, coordenação motora, relação entre potência muscular e peso corporal de uma só vez(Diamond, 2012; Gil \& Araújo, 1999).

O estudo se manteve como intermediário no coeficiente de trabalho e diferentemente dos demais estudos foi utilizada a escala de OMNI-GSE para identificação da intensidade do exercício, denominando em $80 \%$ da escala, apresentando uma alta intensidade. Quando comparado as outras publicações dos autores, manteve-se com a maior intensidade, porém os outros estudos mensuram essa variável em uma repetição máxima. Devido a elevada intensidade, em consequência a frequência semanal foi menor, de apenas duas vezes por semana, tendo uma recuperação maior e para a população idosa é fundamental ter uma recuperação mais extensa quando comparada a jovens adultos.

De acordo com Silva et al.(Silva et al., 2014) esta escala está relacionada a mensuração do esforço físico, envolvendo uma tensão relativamente grande sobre os sistemas musculoesquelético, cardiovascular e respiratório. A escala é um indicador de esforço que tem validade, sendo confiável para monitorar o indivíduo ao exercício, estando relacionada com a frequência cardíaca ou o consumo de oxigênio. A percepção de esforço está associada as categorias: 0,1,3,4,6,8 e 9(Tiggemann, Pinto, Fernando, \& Kruel, 2010).

Observou-se ausência de análise mais minuciosa sobre o controle postural das amostras nos estudos selecionados. A análise do controle postural é feita por meio de cinemetria. A cinemetria avalia o desempenho do sistema de controle postural em condições que envolvem variações de postura, podendo ser de olhos abertos e fechados. É utilizado câmeras para filmagens detectando os movimentos e uma análise biomecânica quantitativa(Mantovani et al., 2014). Para a população idosa esta avaliação é de grande valia, pois há alterações no funcionamento do sistema de controle postural, interferindo na interdependência, execução de exercícios, e atividades diárias. 


\section{Conclusão}

O presente estudo possibilitou uma análise minuciosa sobre os exercícios multiarticulares e monoarticulares e sua influência na capacidade funcional dos idosos. Observou-se que há escassez na literatura a respeito do assunto nessa população. No entanto possibilitou uma especulação de que os exercícios multiarticulares possam ser mais efetivos quando comparado aos exercícios monoarticulares na funcionalidade dos idosos, devido ao recrutamento de mais grupamentos musculares. Dentre a amostra, os estudos que apresentaram valores inferiores do coeficiente de eficiência em relação à funcionalidade, teve menor número ou ausência de exercícios multiarticulares em seu protocolo de treinamento.

Observando-se os resultados do coeficiente de eficiência e de trabalho dos estudos, notou-se que tais resultados podem sofrer influências como o tipo de amostra do estudo (indivíduos ativos ou sedentários), protocolos de treinamento (tempo de estudo, tipos de exercícios, variáveis de volume e intensidade, métodos de treinamento) e a percepção complexa ou não dos testes que avaliam o desempenho funcional. Analisando-se os achados, acredita-se ser possível melhorar a capacidade funcional priorizandoas variáveis volume ou a intensidade com uso de exercícios multiarticulares. Ambas variáveis sendo bem executadas e planejadas com um descanso ativo e recuperação necessária serão eficazes.

Os exercícios monoarticulares também são importantes, pois segundo a literatura, contribuem num possível desequilíbrio muscular, a fim de evitar futuras lesões. Por tanto é necessário um acompanhamento profissional para elaboração de planejamento, com intuito de prescrever exercícios adequados para atender a necessidade do idoso.

A literatura científica sobre a temática ainda é incipiente na população idosa, e salienta-se a necessidade de avaliação postural, por meio de cinemetria, no qual muitos estudos não abordam esse meio de avaliação que é fundamental para essa população.

\section{Financiamento}

Não houve financiamento.

\section{Conflito de interesses}

Não houve conflito de interesses.

\section{Referências}

Albin, I. L. R., Freitas, C. de la R., Teixeira, A. R., Gonçalves, A. K., \& Bós, A. M. P. V. dos S. Â. J. G. (2006). Influência do treinamento de força muscular e de flexibilidade articular sobre o equilíbrio corporal em idosas. Revista Brasileira de Geriatria e Gerontologia, 8(1), 17-26.

Bertani, R. F., Campos, G. D. O., \& Patrizzi, L. J. (2014). Teste de uma repetição máxima em idosos : validade, reprodutibilidade e segurança. EFDeportes.Com Revista Digital, (3), 3-6.

Campinas, U. E. De, Souza, R. De, \& Argento, V. (2010). Benefícios da atividade física na saúde e qualidade de vida do idoso. Trabalho de Conclusão de Curso Apresentado à Graduação Da Faculdade de Educação Física Da Universidade Estadual de Campinas Para Obtenção Do Título de Bacharel Em Educação Física.

César, C. C., Mambrini, J. V. de M., Ferreira, F. R., \& Lima-Costa, M. F. (2015). Capacidade funcional de idosos : análise das questões de mobilidade, atividades básicas e instrumentais da vida diária via Teoria de Resposta ao Item Functional capacity in the elderly: analyzing questions on mobility and basic and instrumental activiti. Caderno de Saúde Pública, 31(5), 931-945.

Coelho, B. dos S., Souza, L. K. de, Roncada, R. B. C., Tiggemann, C. L., \& Dias, C. P. (2014). Comparação da força e capacidade funcional entre idosos praticantes de musculação, hidroginástica e não praticantes de exercícios físicos. Rev. BRas. GeRiatR. GeRontol, $497-504$.

Coggan, A. R., Spina, R. J., King, D. S., Rogers, M. A., Brown, M., Nemeth, P. M., \& Holloszy, J. O. (1992). Histochemical and Enzymatic Comparison of the Gastrocnemius Muscle of Young and Elderly Men and Women. Journal of Gerontology: BIOLOGICAL SCIENCES, 47(3), 71-76.

Das, I., Hormonais, R., Rodrigues, R., Frois, D. S., Roberto, P., \& Gentil, V. (2011). Revista Brasileira de Prescrição e Fisiologia do Exercício. Revista Brasileira de Prescrição e Fisiologia Do Exercício, 473-479.

Diamond, A. (2012). Activities and Programs That Improve Children's Executive Functions. Current Directions in Psychological Science, 21(5), 335-341. https://doi.org/10.1177/0963721412453722 
Fleck, S. J., \& Figueira Junior, A. J. (2003). Treinamento de força para fitness e saúde. In São Paulo: Phorte.

Galvão, T. F., \& Pereira, M. G. (2014). Revisões sistemáticas da literatura: passos para sua elaboração. Epidemiol. Serv. Saúde, 23(1), 183-184. https://doi.org/10.5123/S1679-49742014000100018

Gentil, P., Soares, S., \& Bottaro, M. (2015). Single vs . Multi-Joint Resistance Exercises : Effects on Muscle Strength and Hypertrophy. Asian J Sports Med, 6(2), 2-6. https://doi.org/10.5812/asjsm.24057

Gil, C., \& Araújo, S. De. (1999). Teste de sentar-levantar : apresentação de um procedimento para avaliação em Medicina do Exercício e do Esporte. Revista Brasileira de Medi, 5(5), 179-182.

Kobayashi, H., Koyama, Y., Enoka, R. M., \& Suzuki, S. (2012). A unique form of light-load training improves steadiness and performance on some functional tasks in older adults. Scand J Med Sci Sports, 1-13. https://doi.org/10.1111/j.1600-0838.2012.01460.x

Lima, R. M., Ferreira, C. E. S., Bezerra, L. M. A., Rabelo, H. T., Júnior, L. C. D. S., Santos, E. P. Dos, \& Oliveira, R. J. De. (2012). Estudo de associação entre força muscular e massa magra em mulheres idosas. Rev. Bras. Ciênc. Esporte, 34(4), 985-997.

Lorenzi, M., Araújo, M. De, Fló, C. M., \& Muchale, S. M. (2010). Efeitos dos exercícios resistidos sobre o equilíbrio e a funcionalidade de idosos saudáveis : artigo de atualização Effects of resistance training on balance and functional ability in healthy elderly : an update. Fisioterapia e Pesquisa, 17(3), $277-283$.

Lustosa, L. P., Silva, J. P., Coelho, F. M., Pereira, D. S., Parentoni, A. N., \& Pereira, L. S. M. (2011). Efeito de um programa de resistência muscular na capacidade funcional e na força muscular nos extensores de joelho em idosas pré-frágeis da comunidade: ensaio clínico aleatorizado do tipo crossover. Rev Bras Fisioter, 15(4), 318-324.

Maitin, I. B. (2016). CURRENT medicina e reabilitação: diagnóstico e tratamento. In Porto Alegre: AMGH.

Mantovani, A. M., Chagas, E. F., Claudia, A., Fortaleza, D. S., Federighi, E., Chagas, B., \& Ferreira, A. (2014). Análise do controle postural em diferentes condições por meio de cinemetria. Estud. Interdiscipl. Envelhec., Porto, 19(2), 513-524.

Mcardle, W. D., Katch, F. I., \& Katch, V. L. (1998). Fisiologia do exercício: energia, nutrição e desempenho humano. In 4 ed. Rio de Janeiro: Guanaba Koogan.

Nasser, I., \& Neto, V. G. C. (2017). Treinamento de força com baixas cargas e alto volume para hipertrofia: análise de parâmetros moleculares. Revista Brasileira de Prescrição e Fisiologia Do Exercício, 610-619.

Nathani Amorim Viana, \& Clélia Maria de Sousa Ferreira Parreira. (2013). As contribuições da atividade física regular para apromoção do envelhecimento Monografia Apresentada Ao Curso de Graduação Em Saúde Coletiva, Da Faculdade de Ceilândia Da Universidade de Brasília, Como Requisito Para Obtenção Do Grau de Bacharel Em Saúde Coletiva. Orientadora.

Neto, A. G. de R., Santos, M. S., Silva, R. J. S., Santana, J. M. de, \& Silva-Grigoletto, M. E. Da. (2018). Effects of different neuromuscular training protocols on the functional capacity of elderly women. Rev Bras Med Esporte, 24(2).

Nogueira, S. L., Ribeiro, R. C. L., Rosado, L. E. F. P. L., Franceschini, S. C. C., Ribeiro, A. Q., \& Pereira, E. T. (2010). Fatores determinantes da capacidade funcional em idosos longevos Determinant factors of functional status among the oldest old. Revista Brasileira de Fisioterapia, 14(4).

Orssatto, L. B. da R., Moura, B. M. de, Sakugawa, R. L., Radaelli, R., \& Diefenthaeler, F. (2017). Leg press exercise can reduce functional hamstring/quadriceps ratio in the elderly. Journal of Bodywork \& Movement Therapies. https://doi.org/10.1016/j.jbmt.2017.09.023

Pinheiro, H. A., Pereira, L. C., Santana, F. S. de, Alves, A. T., Fachin-Martins, E., \& Margô Gomes de Oliveira Karnikowski, R. L. de M. (2018). Treinamento de resistência para hipertrofia muscular em idosos Resistance training for muscular hypertrophy in elderly. Fisioterapia Brasil, 19(1), 118-126.

Roncato, M., Galaza, E., Freire, B., Tiggermann, C. L., \& Dias, C. P. (2014). Correlação da força e composição corporal com a capacidade funcional em mulheres idosas. R. Bras. Ci. e Mov., 22(1), 122-130.

Silva, D. D. O., Pazzinatto, M. F., \& Albuquerque, C. E. De. (2014). Influência da preocupação com quedas na mobilidade e na força de reação do solo em idosas durante descida de escada. Scientia Medica, 24(4), 361-367.

Tiggemann, C. L., Pinto, R. S., Fernando, L., \& Kruel, M. (2010). A Percepção de Esforço no Treinamento de Força Perceived Exertion in Strength Training. Revista Brasileira de Medicina Do Esporte, 16, 301-309.

Tracy, B. L., \& Enoka, R. M. (2006). Steadiness Training with Light Loads in the Knee Extensors of Elderly Adults. Official Journal of the American College of Sports Medicine, 735-745. https://doi.org/10.1249/01.mss.0000194082.85358.c4 